\title{
The Role for Advanced Practice Nursing in Canada
}

Sandra MacDonald-Rencz, Executive Director, Office of Nursing Policy, Strategic Policy Branch, Health Canada

Rachel Bard, RN, MA Ed, Chief Executive Officer, Canadian Nurses Association

The delivery of modern health services is a complex activity that increasingly relies on inter-professional collaboration. The different roles of the members of these inter-professional teams may depend not only on traditional job demarcations but also on a division of labour that maximizes efficiency and improves outcomes.

Canadian health policy makers and healthcare managers are continually seeking opportunities to optimize healthcare delivery by modernizing the roles and mix of health professionals, including nurses. Innovations in care are being implemented in response to growing healthcare demands (driven by a rising prevalence of chronic diseases), limited access to physicians (in general or in certain specialties or geographic areas) and tight budget constraints. In addition, in countries where the supply of nurses itself may be an issue, the development of more advanced practice roles may be seen as a way to increase recruitment and retention rates by enhancing career prospects in the profession.

In Canada, discussions about possible extensions of the roles of nurses have taken place in the context of broader efforts to reorganize health service delivery, particularly in the primary care sector and in the development of home- and community-based care options. Two categories of advanced nursing practice roles have emerged to address these needs: nurse practitioners (NPs) and clinical nurse specialists (CNSs). Many NPs practise in primary care, acting as the first contact for people with minor illness, providing routine follow-up for patients with chronic conditions, prescribing drugs and/or ordering tests. Other NPs practise in acute care settings in hospitals or in specialized outpatient settings to provide 
advanced nursing care for patients who are acutely, critically or chronically ill with complex conditions. CNSs tend to work in hospitals, where their responsibilities include conducting research and providing leadership and education to the nursing staff to promote high standards of care and patient safety.

A recently published Organisation for Economic Co-operation and Development report cites Canada and the United States as world leaders in the implementation of advanced practice roles (Delamaire and Lafortune 2010). Canada has been experimenting with and implementing new advanced nursing practice roles for many decades. In the United States, the introduction of NPs, who are responsible for delivering a wide range of services with a high level of autonomy, dates back to the mid-1960s. In many European countries, the development of advanced nursing practice roles is still in its infancy, although some countries, such as France, have recently launched a series of pilot projects to test new models of teamwork between doctors and nurses in primary care and chronic disease management.

Research investments to date have identified key areas of action to further the implementation of advanced practice roles. Progress has also been made in providing a framework that delineates whether an advanced nursing practice role is appropriate in given circumstances. Other research on NP practice tells us how positive the health outcomes are, but it does not provide enough economic analysis. We know even less about the impact of CNS practice in Canada. More research that clarifies the true impact of advanced practice can assist the health system as decision makers grapple with financial constraints and seek to maximize value for money. Disseminating the results of such research is no less important.

A number of very promising innovations in care are being driven by advanced practice nurses in intervening with high-acuity patients who require the coordinated actions of a number of specialized professionals in areas such as neonatology and cardiology. We are seeing a growing number of examples where NPphysician collaboration in primary care (in both NP-led clinics and fee-for-service physician practice) has significantly lowered wait times and improved patient access to care. We are also seeing that, with anticipated changes in the nursing workforce, and as CNSs collaborate with and lead inter-professional teams, the role of the CNS is becoming even more critical for supporting nurses and providing clinical expertise. As the adoption of healthcare models based on inter-professional collaboration becomes more widespread, there exists a unique opportunity to identify niches that can best be filled by advanced practice nurses.

The evolution of advanced practice has had as much to do with enhancing patient-centred care as it has with the expertise required to remain at the cutting edge of clinical and technological advancements. Clinical leadership, support 
for nursing staff and the advancement of research coalesce to produce synergies leading to better outcomes and enhanced patient experiences. As we increase our ability to capture metrics, particularly financial indicators, on the tangible benefits of advanced practice, we will likely see more opportunities created. However, the great variability in CNS specialties makes it more difficult to capture data relevant to these roles.

The papers presented in this issue do an excellent job of identifying and consolidating the various factors that have both enabled and impeded the development and integration of advanced nursing practice in Canada. It is clear from the research that a collaborative approach is needed to implement the recommendations, address outstanding issues, and help build the necessary infrastructure and networks to support nurses working in advanced practice roles. Many of the identified challenges arise from variations in educational requirements and programs, credentialling, legislation and regulation. The continued collaboration among educators, regulatory bodies, policy makers and governments will, undoubtedly, work to address these inconsistencies.

To this end, professional nursing organizations, regulators, educators and researchers have worked with federal, provincial and territorial governments and with research agencies to develop pan-Canadian measures for integrating the NP role in primary care. The Canadian Nurse Practitioner Initiative, supported through the Primary Health Care Transition Fund between 2004 and 2006, has been particularly successful in this regard.

It is also important for nursing leaders to continue to introduce advanced practice nursing roles that align with current and emerging population health and system needs, and to ensure that these roles meet the criteria developed through broader national frameworks.

The key to the future of the healthcare system lies in successfully integrating health professionals into teams that are cohesive, high-performing units. As colleagues and leaders, we join the authors in stressing the importance of all professional groups working together to develop innovative models of care that can address current and emerging healthcare gaps. We encourage you to learn from the papers, which bring new insights into advanced nursing practice in Canada. This information is especially valuable given that much of the unrealized potential surrounding advanced practice can be linked to a lack of clarity in role definition and implementation. Without such research, the development of advanced practice roles would likely continue to experience growing pains and fall short of the tremendous benefits that could be realized. 
Despite the many challenges, the future of advanced nursing practice shows great promise. We will fulfill this potential if we, as nursing leaders, increasingly act in concert to promote clarity and consistency in our collective approach to addressing remaining barriers. Better development and integration of advanced practice roles are crucial to realizing the full contribution of nursing to sustainable, accessible, quality healthcare in Canada.

\section{Reference}

Delamaire, M-L and G. Lafortune. 2010. Nurses in Advanced Roles: A Description and Evaluation of Experiences in 12 Developed Countries. OECD Health Working Paper No. 54. Retrieved July 12, 2010. $<$ www.oecd.org/els/health/workingpapers $>$. 